\title{
Anti-PD-L1 Monoclonal Antibody CK-301
}

National Cancer Institute

\section{Source}

National Cancer Institute. Anti-PD-L1 Monoclonal Antibody CK-301. NCI Thesaurus. Code C151947.

An immunog lobulin G1 (IgG1), human monoclonal antibody directed against the immunosuppressive lig and programmed cell death-1 ligand 1 (PD-L1; cluster of differentiation 274; CD274), with potential immune checkpoint inhibitory and antineoplastic activities. Upon administration, anti-PD-L1 monoclonal antibody CK-301 specifically targets and binds to PD-L1, blocking its binding to and activation of its receptor prog rammed cell death protein 1 (PD-1; PDCD1; CD279; programmed death-1). This reverses T-cell inactivation caused by PD-1/PD-L1 signaling and enhances the cytotoxic T-lymphocyte (CT L)-mediated anti-tumor immune response against PD-L1expressing tumor cells. PD-L1 is overexpressed by many human cancer cell types. PD-L1 binding to PD-1 on T-cells suppresses the immune system and results in immune evasion. PD-1, a transmembrane protein belonging to the immunoglobulin superfamily expressed on activated T-cells, is a negative regulator of the immune system that limits the expansion and survival of CD8-positive T-cells. 